\title{
Computational Modelling of Water Oxidation Catalysts
}

Joaquín Soriano-López ${ }^{1}$, Wolfgang Schmitt ${ }^{1}$ and Max García-Melchor ${ }^{1, *}$

${ }^{1}$ School of Chemistry and CRANN/AMBER Nanoscience Institute, Trinity College, The University of Dublin, College Green, Dublin 2, Ireland

* To whom correspondence should be addressed: garciamm@tcd.ie

\section{Keywords}

Oxygen evolution reaction, water oxidation, density functional theory, electrocatalysis, reaction descriptors, reaction mechanisms.

\begin{abstract}
In this opinion, we review the state-of-the-art in the modelling of the water oxidation reaction catalysed by homogeneous and heterogeneous systems. We start by introducing the potential and current limitations in the development of energy conversion technologies based on this process, followed by a brief description of the two main proposed reaction mechanisms. We next present an overview of the different theoretical approaches adopted to describe this reaction, and summarise the most recent computational works devoted specifically to the investigation of the O$\mathrm{O}$ bond formation step. This latter part also includes a review of the advances in the modelling of electrochemical energy barriers. The chemical descriptors proposed to rationalise the OER activity and the theoretical methods developed to account for solvent effects, are also reviewed. Finally, we present a selection of theoretical studies reported over the last two years to illustrate the descriptive and predictive power of computational methods.
\end{abstract}




\section{Introduction}

Due to the depletion of fossil fuels and environmental concerns over their use, it is critical that we cut our energy dependence on fossil fuels and develop technologies that utilise renewable energy sources.[1] The latter, however, are inherently intermittent, which brings about the necessity of storing the electricity generated from these sources in the form of high-energy-density chemical bonds, such as $\mathrm{H}_{2}$, by means of electrochemical water splitting.[2,3] While this sustainable technology has a tremendous potential, its development is currently hampered by the lack of efficient, inexpensive, and robust electrocatalysts for the water oxidation reaction, also known as the oxygen evolution reaction (OER):

$$
2 \mathrm{H}_{2} \mathrm{O}_{(\mathrm{l})} \rightarrow \mathrm{O}_{2(\mathrm{~g})}+4 \mathrm{H}^{+}+4 \mathrm{e}^{-} \quad \mathrm{E}^{\circ}=1.23 \mathrm{~V} \text { vs RHE }
$$

In principle, an ideal OER electrocatalyst should be a material that (i) yields efficient oxygen conversion rates at the thermodynamic potential while delivering high current densities; (ii) is based on earth-abundant elements; and (iii) exhibits long-term stability under strong oxidizing conditions, preferably in acidic media, where proton exchange membranes show optimal performance. Up to date, only the noble metal oxides $\mathrm{IrO}_{2}$ and $\mathrm{RuO}_{2}$ (or derivatives of these) have been able to meet most of these stringent criteria,[4] but their scarcity and prohibitive price have hindered their further use in large-scale applications. First-row transition metal oxides have recently emerged as an attractive alternative, exhibiting good OER performances in strong alkali media. $[5,6]$ In this case, the main drawback is that only few oxides can preserve their high activity at neutral or acidic $\mathrm{pH} .[7-9]$

To develop more efficient OER electrocatalysts, considerable efforts have been devoted to devise ways to increase the number of catalytic active sites and their intrinsic activity. Particularly, significant advances have been made by taking inspiration from the oxygen evolution complex in the photosystem II,[10,11] adding dopants or promoters,[12,13] nanostructuring catalysts, $[14,15]$ and introducing confined environments.[16,17] In addition, approaches to boost the electrocatalytic activity of molecular catalysts have included ligand functionalization[18] and electrode modification.[19,20] Despite the substantial progress achieved with the implementation of these strategies, further enhancement of OER electrocatalysts may require a detailed understanding of this rather complex process. In this regard, density functional theory (DFT) calculations have been proven to be very valuable, for example, in the elucidation of the underlying OER mechanisms at the atomic level, the rationale between structure and OER activity, and the proposal of reaction descriptors to guide the design of better OER catalysts in a reasonable period of time.

The state-of-the-art of these and other relevant aspects related to the modelling of OER electrocatalysts are reviewed in this opinion, with special focus on DFT works published in the past two years. For an overview of experimental studies on the OER, we refer the reader to the recent opinion by Lyons et al.[21] 


\section{Modelling of the OER mechanism}

The overall OER process involves four elementary proton-coupled electron transfer (PCET) steps, where the proton and electron transfer can occur either in a concerted or sequential fashion.[22,23] Typically, the OER mechanism starts with two PCET to yield a metal-oxo species, which subsequently undergoes $\mathrm{O}-\mathrm{O}$ bond formation (Figure 1). This latter step has been proposed to occur through two main reaction pathways, namely the water nucleophilic attack (WNA) and the interaction of $2 \mathrm{M}=\mathrm{O}$ entities (I2M). As shown in Figure 1, the WNA mechanism involves the attack of a solvent water molecule together with a PCET resulting in a hydroperoxo intermediate, which finally evolves molecular oxygen via another PCET. On the other hand, the formation of the $\mathrm{O}-\mathrm{O}$ bond in the I2M mechanism takes place between two metal-oxo units, leading to a peroxo species that eventually produces $\mathrm{O}_{2}$ and regenerates the catalyst.

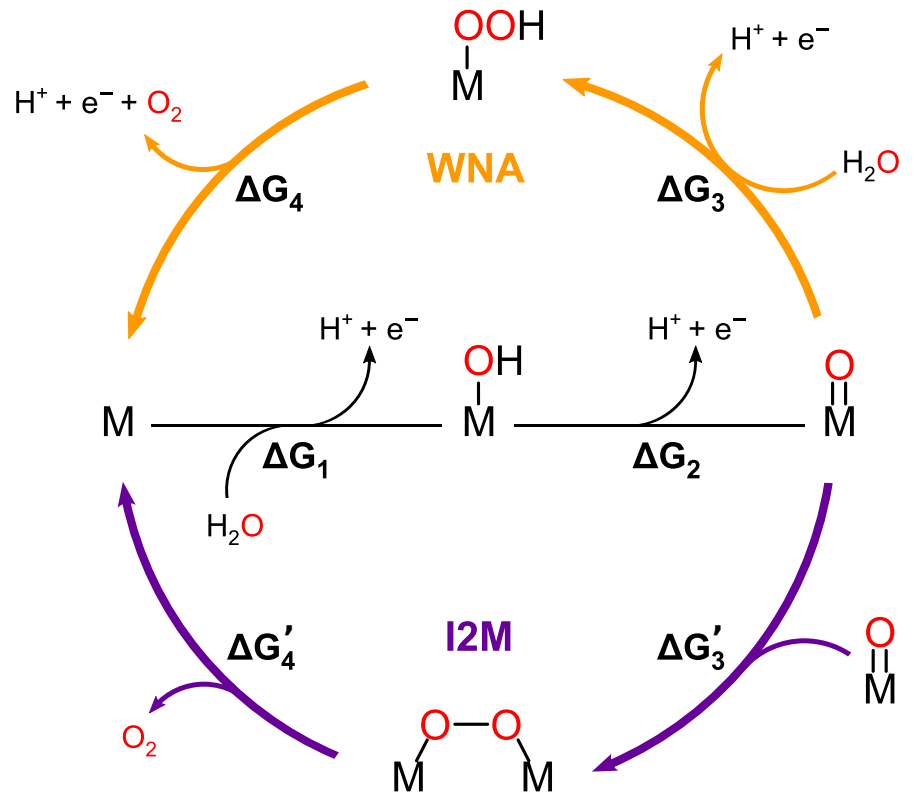

Figure 1. Catalytic cycle of the two main reaction mechanisms proposed for the OER.

The modelling of the OER mechanism with homogeneous catalysts has been mostly centred on the study of the chemical speciation and the O-O bond formation step. Extensive works on the chemical speciation have consisted in the construction of energy diagrams where both concerted and sequential PCET events are considered. These diagrams, commonly known as "square diagrams", have allowed the elucidation of the catalytic species involved in the O-O bond formation of numerous molecular OER catalysts.[24-26] The details of the methodology employed in the computation of square diagrams, as well as other examples of applications, can be found in a recent review article by Truhlar and co-workers.[27]

For a wealth of homogeneous catalytic systems, the O-O bond formation has been shown to proceed through the WNA mechanism, although examples of catalysts operating via the I2M process have also been reported based on second order kinetic experiments and DFT studies.[28] One recent example is the theoretical work by Siegbahn,[29] where both WNA and I2M pathways were discussed for the $\mathrm{Mn}_{4} \mathrm{CaO}_{5}$ cluster of the photosystem II. Therein, two different radical metal- 
oxyl sites were identified to be susceptible to the attack of a solvent water, although the lowest activation barrier found for this step was significantly higher than that calculated via the I2M pathway.

DFT studies on the complete catalytic cycle for homogeneous OER catalysts have also been reported, although they are scarce. Cramer and co-workers[30] recently reviewed the overall OER mechanism for a series of Ru and earth-abundant complexes, while Poblet et al.[31*] carried out a thorough DFT study on the OER mechanism for a cobalt-based polyoxometalate cluster $\left[\mathrm{Co}_{4}\left(\mathrm{H}_{2} \mathrm{O}\right)_{2}\left(\mathrm{PW}_{9} \mathrm{O}_{34}\right)_{2}\right]^{10-}\left(\mathrm{PCo}_{4}\right)$. This latter work included various square diagrams and suggested that the $\mathrm{O}-\mathrm{O}$ bond formation takes place from a $\mathrm{Co}^{3+}$-oxyl radical through the WNA pathway.

In the case of heterogeneous catalysts, computational studies have shown that kinetic barriers associated to PCET steps are typically of the order of $0.2 \mathrm{eV}$.[32] Because this energy barrier may be surmountable at room temperature, most of the DFT studies reported thereafter have focused on the thermodynamic description of the OER. With this assumption, the relative Gibbs energies for the four elementary steps of the WNA mechanism can be expressed as:

$\Delta \mathrm{G}_{1}=\Delta \mathrm{G}_{\mathrm{HO}^{*}}-\Delta \mathrm{G}_{\mathrm{H}_{2} \mathrm{O}}-e \mathrm{U}+k_{\mathrm{B}} \mathrm{T} \ln a_{\mathrm{H}^{+}}$

$\Delta \mathrm{G}_{2}=\Delta \mathrm{G}_{\mathrm{O}^{*}}-\Delta \mathrm{G}_{\mathrm{HO}^{*}}-e \mathrm{U}+k_{\mathrm{B}} \mathrm{T} \ln a_{\mathrm{H}^{+}}$

$\Delta \mathrm{G}_{3}=\Delta \mathrm{G}_{\mathrm{HOO}^{*}}-\Delta \mathrm{G}_{\mathrm{O}^{*}}-\Delta \mathrm{G}_{\mathrm{H}_{2} \mathrm{O}}-e \mathrm{U}+k_{\mathrm{B}} \mathrm{T} \ln a_{\mathrm{H}^{+}}$

$\Delta \mathrm{G}_{4}=\Delta \mathrm{G}_{\mathrm{O}_{2}}-\Delta \mathrm{G}_{\mathrm{HOO}^{*}}-e \mathrm{U}+k_{\mathrm{B}} \mathrm{T} \ln a_{\mathrm{H}^{+}}$

where * refers to a surface active site, $\mathrm{HO}^{*}, \mathrm{O}^{*}$, and $\mathrm{HOO}^{*}$ are adsorbed OER intermediates, and the term $e \mathrm{U}$ accounts for the applied potential.

To calculate the relative Gibbs energies shown in equations (2)-(5), two assumptions have been typically made. First, the Gibbs energy of the overall reaction is fixed to the experimental value of $4.92 \mathrm{eV}$ to avoid the introduction of the error in the description of molecular oxygen with DFT methods. Hence, the term $\Delta \mathrm{G}_{\mathrm{O}_{2}}-\Delta \mathrm{G}_{\mathrm{HOO}^{*}}$ in equation (5) may be rewritten as $4.92-\Delta \mathrm{G}_{\mathrm{HOO}^{*}}$. The second assumption is that the Gibbs energy of the proton-electron couple can be computed as half of the Gibbs energy of gas hydrogen, as these species in solution are in equilibrium at $0.00 \mathrm{~V}$. This latter approach is widely known as the computational hydrogen electrode model.[33]

From the above relative energies, the potential-determining step (PDS), i.e. the thermodynamically most energy demanding process, is given by:

$\Delta \mathrm{G}_{\mathrm{PDS}}=\max \left\{\Delta \mathrm{G}_{1}, \Delta \mathrm{G}_{2}, \Delta \mathrm{G}_{3}, \Delta \mathrm{G}_{4}\right\}$

The theoretical overpotential can be then calculated by subtracting the thermodynamic oxidation potential of water to the calculated Gibbs energy of the PDS:

$\eta_{\text {theor }}=\left(\Delta \mathrm{G}_{\mathrm{PDS}} / e\right)-1.23 \mathrm{~V}$

Although this methodology might seem oversimplified, other results which will be highlighted later in this opinion have successfully employed this approach to rationalise the activity of OER 
electrocatalysts, identify the local geometry of active sites, and even guide the design of more efficient catalysts.

Similarly to homogeneous catalysts, the I2M mechanism for heterogeneous systems has been much less studied.[34,35] In this context, it is worth highlighting the recent DFT+U investigation by Carter et al.[36] on a nickel oxyhydroxide (i.e. $\beta$ - NiOOH), where two variants of the I2M mechanism were predicted to be favoured over the WNA pathway. Another intriguing finding was recently reported in a combined experimental and theoretical work by Shao-Horn and coworkers,[37] which showed that lattice oxygens atoms in some perovskites may also participate in the $\mathrm{O}-\mathrm{O}$ bond formation.

The computation of reaction barriers of PCET events on the surface of heterogeneous catalysts has also received some attention, although it still represents one of the biggest challenges in the modelling of electrochemical processes. This stems from the fact that simulations are usually performed keeping the number of electrons constant, i.e. constant charge, whereas electrochemical reactions are carried out at constant potential. To overcome this issue, one can in principle increase progressively the size of the cell, as the change in interface charge density during the PCET step decreases with the size of the cell and becomes zero by extrapolation to the infinite-size cell limit (Figure 2). However, while this cell extrapolation method might be suitable to model simple reaction mechanisms like the hydrogen reduction/oxidation reaction,[38,39] it becomes computational prohibitively expensive for more complicated processes such as the OER.

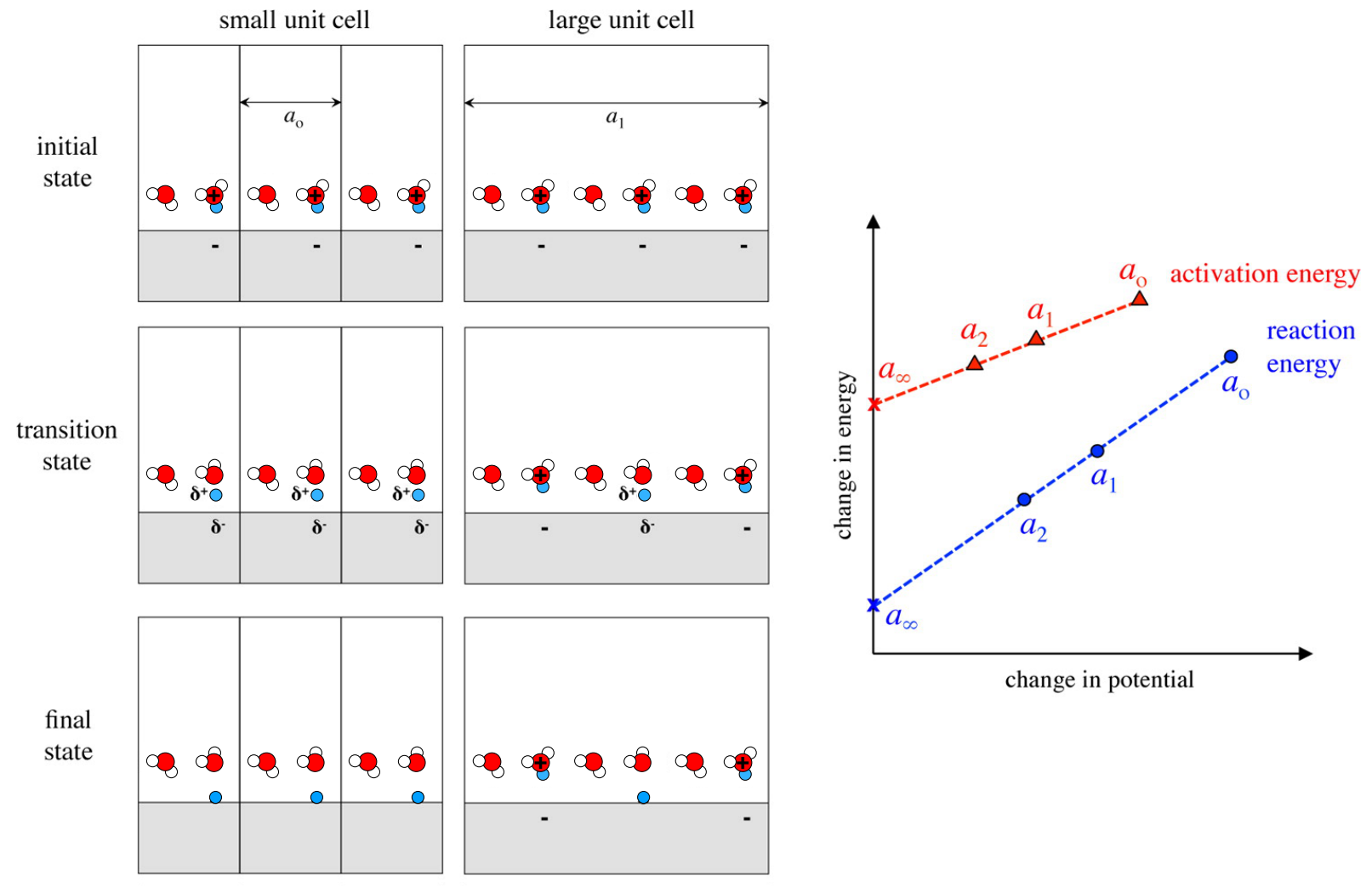

Figure 2. Left: Schematic showing the proton transfer (highlighted in blue) to a solid surface in unit cells of different size, $a_{o}$ and $a_{l}$. Right: Extrapolation of reaction and activation energies 
calculated at different cell sizes $a_{i}$ and extrapolated to that at infinite cell size $a_{\infty}$, the constant potential limit. Adapted with permission from Ref. [40]. Copyright (C 2015 American Chemical Society.

Recently, Chan and Nørskov developed a method to compute the electrochemical reaction energetics at constant potential by means of "charge-extrapolation" of constant charge calculations using Bader analysis (Figure 2).[40*] It assumes that, in a charge transfer event, the chemical and electrostatic contributions to the reaction energy can be calculated separately, and that the former can be described by a simple capacitor model. Hence, the computation of electrochemical barriers at constant potential only requires the calculation of the activation barrier at a given potential and the corresponding surface charge at the initial, transition, and final states. In a follow-up work, the authors applied this model to evaluate the potential dependence of the energy barriers of various proton-electron transfer reactions, and conclude that this dependence is governed by the partial charge transferred in the transition state.[41]

More recently, Goddard III and co-workers used the Joint Density Functional Theory (JDFTx) method[42] to automatically adjust the number of electrons of the system at a fixed electrochemical potential and compute the Gibbs energy barriers for the OER reaction catalysed by $\operatorname{IrO}_{2}(110)$.[43] Interestingly, the energy of the transition state associated to the rate-determining water dissociation step was found to depend linearly on the applied potential, contrary to what calculations at constant charge predicted. Furthermore, some steps that were thermodynamically favourable resulted to be kinetically less favourable.

\section{Reaction descriptors for the OER}

The thermodynamic picture of the OER mechanism has enabled the proposal of physicochemical properties or chemical descriptors with the aim to explain and predict the performance of OER catalysts at a reduced computational cost. In early works by Rossmeisl and Nørskov, the binding energies of the different OER intermediates were employed to reason the observed catalytic activity of various metal and metal oxide surfaces.[44,45] Importantly, these binding energies were found to be strongly correlated, which led Koper[46] to show that the $\mathrm{HO}^{*}$ and $\mathrm{HOO}^{*}$ binding energies follow a linear scaling relation with a slope of approximately 1 and an intercept of 3.2 (Figure 3). Hence, the energy difference between these two intermediates seemed to be fixed to a constant value of $3.2 \mathrm{eV}$ regardless of the considered catalyst. An important implication of this relation is that the OER activity of almost any catalyst can be assessed by simply computing the difference between the Gibbs adsorption energies of the $\mathrm{O}^{*}$ and $\mathrm{HO}^{*}$ intermediates, $\Delta \mathrm{G}_{\mathrm{O}^{*}}-\Delta \mathrm{G}_{\mathrm{HO}^{*}}$, widely known as the OER descriptor.[47] The graphical representation of this descriptor versus the theoretical overpotential leads to a volcano plot (Figure 3), where the most active catalysts lie on the top. The maximum activity, however, is limited by the existing scaling relationship between the $\mathrm{O}^{*}$ and $\mathrm{HOO}^{*}$ intermediates. More specifically, the difference between the $3.2 \mathrm{eV}$, predicted by the scaling, and the $2.46 \mathrm{eV}$, expected for an ideal catalyst, renders a minimum theoretical overpotential of $0.37 \mathrm{~V}$. This "overpotential wall" is in very good agreement with the reported experimental overpotentials,[4] which reinforces the predicting power of this OER descriptor. 

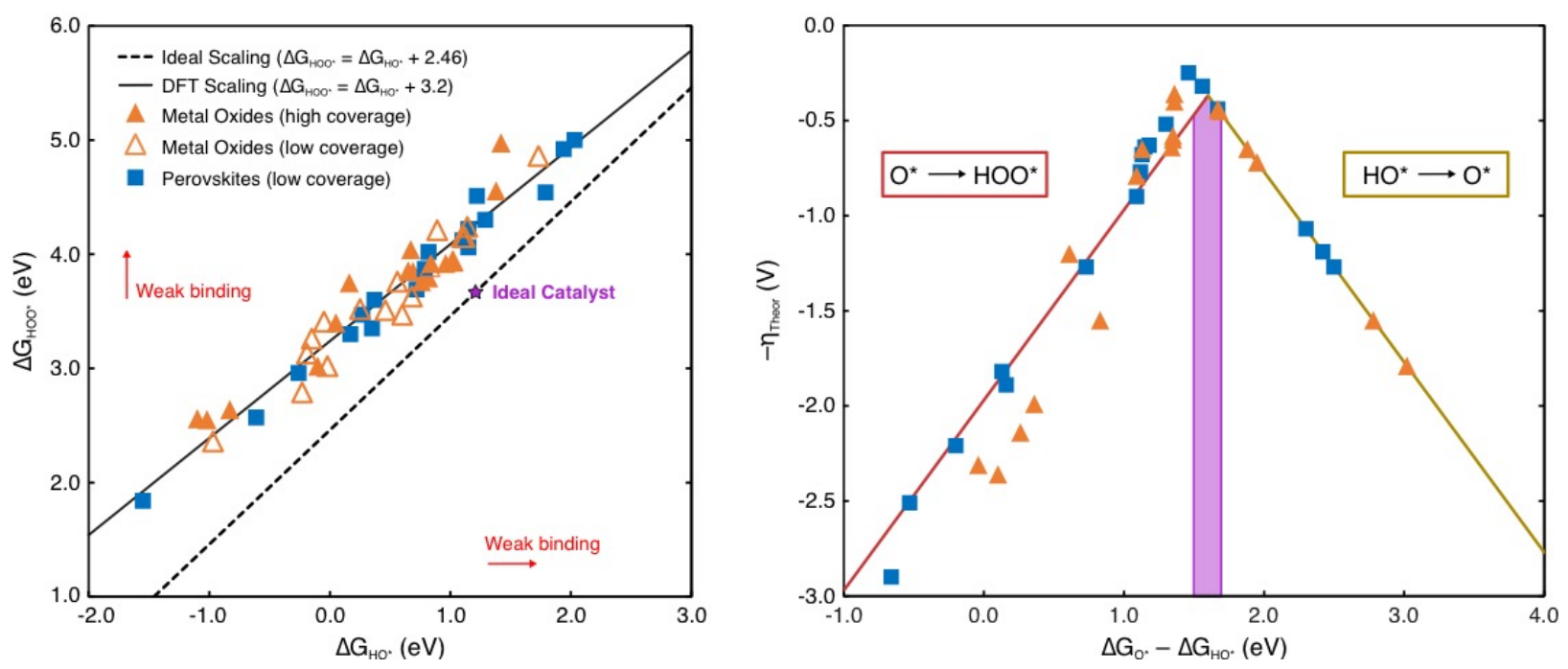

Figure 3. Left: $\mathrm{HO}^{*}$ and $\mathrm{HOO}^{*}$ scaling plot for several metal oxides and perovskites. Right: 2Dvolcano representation of the OER descriptor, $\Delta \mathrm{G}_{\mathrm{O}^{*}}-\Delta \mathrm{G}_{\mathrm{HO}^{*}}$, versus the calculated theoretical overpotential for some of the metal oxides and perovskites shown on the left plot. Data points have been taken from Ref. [33]. Full (empty) orange triangles correspond to metal oxides in a high (low) coverage regime, whereas blue squares correspond to perovskites in a low coverage regime.

Besides this OER descriptor, many other descriptors have been proposed to explain the observed experimental trends. For instance, Shao-Horn recently employed quantitative structure-activity relationships (QSARs) to elucidate which of the 14 studied descriptors yield more reliable predictions of the OER activity of 101 different perovskites. [48*] The authors concluded that the descriptors based on the metal-oxygen bond strength can be grouped in five different families: metal-oxygen covalency, transition-metal electron occupancy - these first two have the strongest influence -, electrostatics, structure, and exchange interactions. In addition, it was found that the number of $d$ electrons and the charge-transfer energy play the most important roles. In a posterior study, Van Voorhis demonstrated that the Sabatier principle based on periodic trends of reactivity can successfully predict the activity of a homo-metallic two-centre metal oxide model system.[49] However, this analysis was shown to oversimplify the activity trends observed for heterobimetallic systems. Toroker also proposed the ionicity of the metal-oxygen bond as a descriptor of the photocatalytic activity of $\beta$-NiOOH.[50] The advantage of using of this descriptor is that ionicity is a bulk property, which avoids the computation of reaction intermediates thus enabling the fast screening of catalysts by a simple Bader charge analysis. Other recent studies that proposed the metal-oxygen bond strength as a descriptor are those by Aizawa et al.[51] and Xia et al.[52] 


\section{Modelling of solvent effects in the OER}

The modelling of chemical reactions occurring at electrode surfaces has been commonly limited to the study of the solid-gas interface. However, solvent is known to play a major role in electrochemistry by influencing the stability of reaction intermediates and the morphology of the electrode surface, among other effects. Nowadays, thanks to the exponential growth in supercomputing performance and the development of more advanced theoretical methods, the study of solvent effects in more complex systems has become possible. The two main approaches to introduce the effect of the solvent are by means of implicit (continuum) and explicit (atomistic) solvation models.

In the implicit approach, the solvent is treated as a parametrized dielectric continuum medium surrounding the solute, which interacts self-consistently with the solvent. Perhaps the most employed continuum method is the polarizable continuum model (PCM),[53] although several variants of it have been also developed, such as the conductor-like polarizable continuum model (CPCM), the solvent model density (SMD), and the conductor-like screening model (COSMO).[54]

Despite having been established for years, the implementation of implicit solvation models for periodic systems have not been available until recently. One of the developed models is the socalled VASPsol by Mathew et al.,[55] which allows the introduction of solvent effects in the modelling of molecules and solid surfaces. This method has been recently extended to describe electrode/electrolyte interfaces and electrochemical reactions.[56] A similar solvation model was developed by Garcia-Ratés and López, where the electrostatic effect caused by the introduction of the solvent was efficiently solved by means of multigrid methods. This method, named VASPMultigrid Continuum Model, was proposed to reduce the computational cost by means of a fast and efficient method for the product of sparse matrices.[57*] Lately, Sundararaman and Schwarz have revised two solvation models for the description of charged metal electrodes that take into account the ionic response into the overall energetics of the continuum approach.[58]

In the explicit approach, solvent molecules are included into the gas phase calculation, which increases the degrees of freedom, and accordingly, the computational cost. Further complications arise due to the large pool of possible configurations of the water molecules and the minimum number of water molecules required for the accurate description of the solvent, which is not trivial.

The effect of adding explicit solvent on the OER energetics of defect-free rutile structured oxide (110) surfaces of $\mathrm{TiO}_{2}, \mathrm{RuO}_{2}$ and $\mathrm{IrO}_{2}$, was recently addressed by Siahrostami and Vojvodic.[59] Therein, water molecules were found to form chains on the oxide surfaces, which affected the OER reactivity by changing the PDS. Particularly, this step varied from the formation of $\mathrm{HOO}^{*}$, computed in vacuum, to the formation of $\mathrm{O}^{*}$, computed with the explicit solvent. This observation was attributed to the high energy required to break the hydrogen bond between the $\mathrm{HO}^{*}$ intermediate and an explicit water molecule. As a result, the overpotential for $\mathrm{TiO}_{2}$ increased compared to that calculated in the absence of explicit water, while for $\mathrm{IrO}_{2}$ and $\mathrm{RuO}_{2}$ it had a minor influence. 
Recently, Nørskov et al. employed the global minimization algorithm called minima hopping to evaluate the OER energetics for a rutile $\mathrm{IrO}_{2}(110)$ surface using two different coverages, i.e. $\mathrm{OH}$ and $\mathrm{O}$, and in the presence of up to 3 water bilayers.[60] Interestingly, only the first two bilayers appeared to have a direct effect on the OER energetics, which suggests that this model should be enough to accurately compute the reaction energies and barriers. In addition, the strongest solvent effect was found to be on the binding energies of OER intermediates that are capable to donate hydrogen bonds, i.e. $\mathrm{HO}^{*}$ and $\mathrm{HOO}^{*}$, whereas the effect on the $\mathrm{O}^{*}$ binding was lesser. Consequently, a major stabilization of ca. $0.4 \mathrm{eV}$ was observed for the $\mathrm{HOO}^{*}$ intermediate on an oxygen covered surface, which resulted in a decrease of the $\mathrm{HO}^{*} / \mathrm{HOO}^{*}$ scaling of ca. $0.3 \mathrm{eV}$.

\section{Beyond the modelling of the OER mechanism}

Besides the investigation of the OER mechanism, computational studies have proven to be very valuable, for example, for the reasoning of the enhanced activity upon catalyst modification, the elucidation of the active phase of catalysts, or the design of more efficient electrocatalysts. In the following, we present a selection of DFT studies reported within the last two years that have successfully addressed one of these issues, which otherwise are very difficult to elucidate by means of experimental techniques.[61,62]

In addition to the study of the OER mechanism with $\mathrm{PCo}_{4}$, Poblet and co-workers investigated the origin of the improved stability and reactivity of the isostructural $\left[\mathrm{Co}_{4}\left(\mathrm{H}_{2} \mathrm{O}\right)_{2}\left(\mathrm{VW}_{9} \mathrm{O}_{34}\right)_{2}\right]^{10-}\left(\mathrm{VCo}_{4}\right)$ polyoxometalate cluster.[31*] The computation of the reaction mechanism with $\mathrm{VCo}_{4}$ showed a higher oxidation potential for the first PCET event and a reduction in the activation energy for the WNA pathway. The authors ascribed both effects to the decrease in the molecular orbital energies due to the orbital-coupling between vanadium and cobalt atoms.

Another excellent example is the joint theoretical and experimental study by Vojvodic and Sargent et al. in which DFT $+\mathrm{U}$ calculations were employed to guide the design of an all earth-abundant material that outperformed the state-of-the-art OER catalysts reported in alkali media.[63*] Starting with the modelling of the pure phases $\beta-\mathrm{CoOOH}, \gamma-\mathrm{FeOOH}, \mathrm{WO}_{3}$, and by means of simple linear interpolation arguments, a near-optimal $\mathrm{HO}^{*}$ binding and a low theoretical overpotential was predicted for a ternary $\mathrm{Fe}, \mathrm{W}$-doped $\mathrm{CoOOH}$ (Figure 4). This ternary oxyhydroxide was subsequently prepared via a sol-gel synthetic route, providing in alkali conditions a record overpotential of $191 \mathrm{mV}$ at $10 \mathrm{~mA} / \mathrm{cm}^{2}$. It is worth highlighting that the stability of this catalyst was also remarkable, maintaining a potential of $c a$. $1.4 \mathrm{~V}$ vs RHE while delivering a constant current density of $30 \mathrm{~mA} / \mathrm{cm}^{2}$ for more than 500 hours.

The same year, Vojvodic, Jaramillo et al. reported the dramatically enhanced activity of $\mathrm{NiO}_{\mathrm{x}}$ by using cerium as dopant and gold as support.[64*] In this case, DFT calculations shed light on the local environment of the OER active sites and the electronic and geometric effects on the OER performance. More specifically, simulations revealed that the incorporation of Ce modifies the local electronic environment of $\mathrm{NiO}_{x}$, leading to a more favourable OER energetics. On the other hand, $\mathrm{Au}$ was suggested to facilitate the access to highly active under-coordinated sites. Altogether, DFT calculations pointed to the synergistic effect of these three components (i.e. Ce- 
doping, Au-support, and under-coordinated sites) as the main responsible for the outstanding performance of this catalyst.
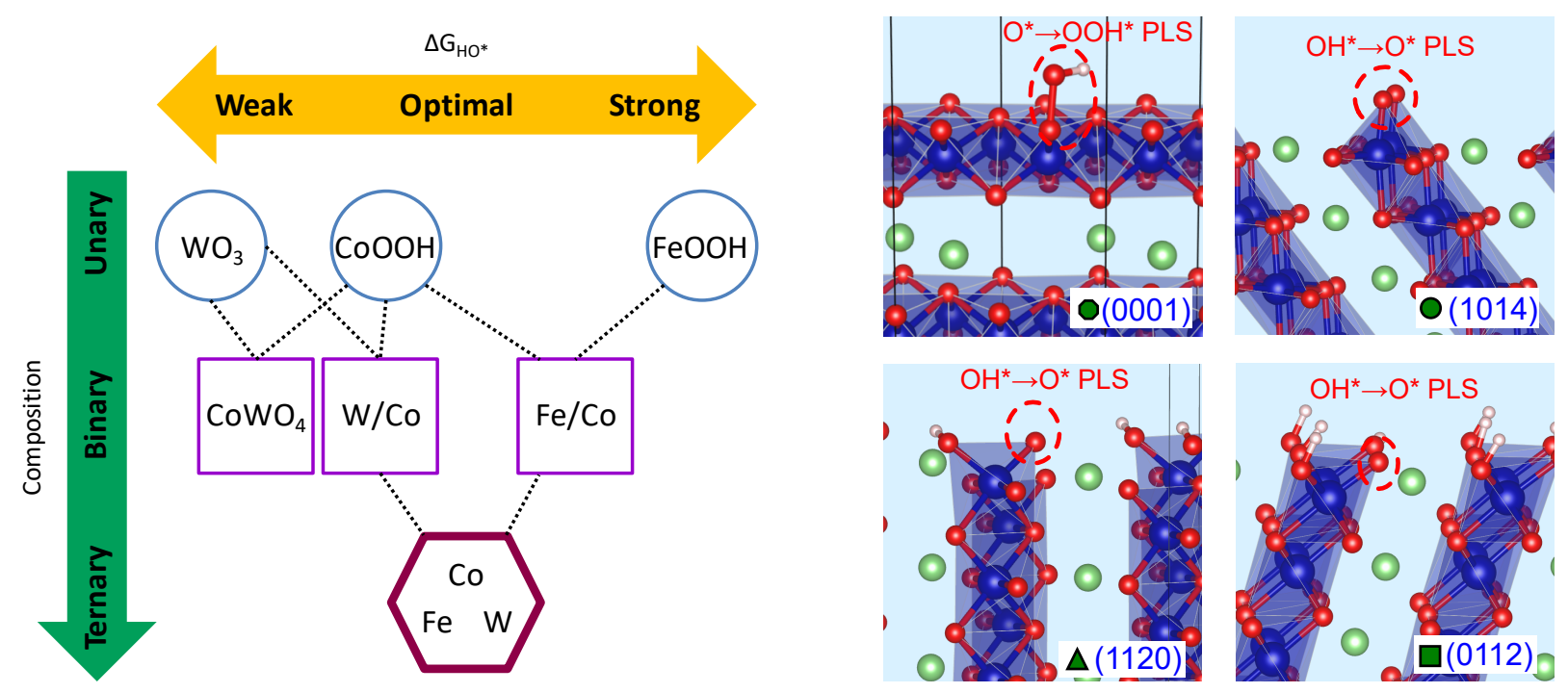

Figure 4. Left: Change in $\mathrm{HO}^{*}$ Gibbs binding energy as a function of chemical composition obtained by interpolation between the calculated pure phases: $\mathrm{WO}_{3}(001), \beta$-CoOOH $(01-12), \gamma-$ $\mathrm{FeOOH}(010)$, and $\mathrm{CoWO}_{4}(010)$. Adapted with permissions from Ref. [63*]. Copyright (C) 2016 The American Association for the Advancement of Science and Copyright Clearance Center. Right: $\mathrm{Li}_{0.5} \mathrm{CoO}_{2}$ surface structures considered in Ref. [65*]. OER intermediates at the end of a potential-limiting step (PLS) are highlighted. Adapted with permissions from Ref. [65*]. Copyright (C) 2017 American Chemical Society.

An important contribution to the elucidation of the catalytically active phase of an OER catalyst was recently reported by Bajdich, Cui and co-workers. $\left[65^{*}\right]$ Therein, DFT $+\mathrm{U}$ calculations were conducted to rationalise the change in the OER activity observed experimentally for a series of lithium cobalt oxide surfaces as a function of the lithium content. Calculations showed that the (0001) surface was not active regardless of the lithium content. Conversely, the overpotential for the (01-12) and (11-20) surfaces was found to decrease after delithiation, whereas it increased for the (10-14) surface. Based on these results, the authors concluded that the (01-12) and (11-20) surfaces are responsible for the enhanced OER activity due to an increase of the $\mathrm{Co}^{4+}$ species after delithiation. These findings were also supported by electrochemical experiments.

The active phase of an $\mathrm{IrO}_{\mathrm{x}} / \mathrm{SrIrO}_{3}$ formed after strontium leaching from a $\mathrm{SrIrO}_{3}$ thin film under electrochemical conditions was also unravelled by means of DFT calculations in a combined theoretical and experimental work by Jaramillo et al.[66*] Importantly, this "unknown" phase displayed in acidic media an overpotential of only $290 \mathrm{mV}$ at $10 \mathrm{~mA} / \mathrm{cm}^{2}$, which represents one of the best OER catalysts reported in these conditions. Based on the calculated theoretical overpotentials for several different $\mathrm{IrO}_{\mathrm{x}} / \mathrm{SrIrO}_{3}$ structures, the authors suggested that the leaching of $\mathrm{Sr}^{2+}$ stabilises the anatase phase of $\mathrm{IrO}_{\mathrm{x}}$, which exhibits a lower overpotential than the more stable rutile phase, in agreement with experiments. 
Finally, DFT calculations by García-Melchor and Vojvodic et al.[67*] showed that a dimeric model Ir complex immobilised on an $\mathrm{IrO}_{2}(110)$ surface displays a comparable OER activity than the isolated dimer. This work also indicated that molecular OER catalysts can be strongly attached to an oxide surface, which suggests that the resulting "hybrid" materials might benefit from the high activity of homogeneous catalysts and the high stability of heterogeneous systems.

\section{Concluding Remarks}

Remarkable progress in the molecular-level understanding of the OER process has been made over the past few years thanks to the development of more sophisticated DFT methods and the exponential growth in computing power. Also important has been the thermodynamic description of the OER, which has allowed the proposal of chemical descriptors to explain the activity of a vast number of materials and even tailor more efficient ones. Importantly, the accuracy of these predictions has rapidly increased due to the considerable efforts made in developing theoretical models to account for solvent effects, which are known to play an essential role. Yet, the accurate description of reaction barriers of PCET events at a reasonable computational cost remains still challenging. Nonetheless, some interesting approaches have been adopted to describe the electrode/electrolyte interface at a constant applied potential. These strategies are very promising and may provide researchers with valuable information about the reaction kinetics at electrode surfaces. Further advances in this field will be inexorably linked to the increase in computing performance and the development of more advanced experimental techniques.

\section{Acknowledgements}

We gratefully thank the School of Chemistry of Trinity College Dublin, the Science Foundation Ireland (SFI; 13/IA/1896), and the European Research Council (CoG 2014-647719) for financial support. The DJEI/DES/SFI/HEA Irish Centre for High-End Computing (ICHEC) is also acknowledged for the provision of computational facilities and support.

\section{References}

[1] Z. Yang, J. Zhang, M.C.W. Kintner-Meyer, X. Lu, D. Choi, J.P. Lemmon, J. Liu, Electrochemical energy storage for green grid, Chem. Rev. 111 (2011) 3577-3613.

[2] Z.W. Seh, J. Kibsgaard, C.F. Dickens, I. Chorkendorff, J.K. Nørskov, T.F. Jaramillo, Combining theory and experiment in electrocatalysis: Insights into materials design, Science 355 (2017) eaad4998.

[3] N.-T. Suen, S.-F. Hung, Q. Quan, N. Zhang, Y.-J. Xu, H.M. Chen, Electrocatalysis for the oxygen evolution reaction: recent development and future perspectives, Chem. Soc. Rev. 46 (2017) 337-365.

[4] C.C.L. McCrory, S. Jung, I.M. Ferrer, S.M. Chatman, J.C. Peters, T.F. Jaramillo, Benchmarking hydrogen evolving reaction and oxygen evolving reaction electrocatalysts 
for solar water splitting devices, J. Am. Chem. Soc. 137 (2015) 4347-4357.

[5] J.R. Galán-Mascarós, Water oxidation at electrodes modified with earth-abundant transition-metal catalysts, ChemElectroChem. 2 (2015) 37-50.

[6] B.M. Hunter, H.B. Gray, A.M. Müller, Earth-abundant heterogeneous water oxidation catalysts, Chem. Rev. 116 (2016) 14120-14136.

[7] M.W. Kanan, D.G. Nocera, In situ formation of an oxygen-evolving catalyst in neutral water containing phosphate and $\mathrm{Co}^{2+}$, Science 321 (2008) 1072-1075.

[8] I. Zaharieva, P. Chernev, M. Risch, K. Klingan, M. Kohlhoff, A. Fischer, H. Dau, Electrosynthesis, functional, and structural characterization of a water-oxidizing manganese oxide, Energy Environ. Sci. 5 (2012) 7081-7089.

[9] R. Frydendal, E.A. Paoli, I. Chorkendorff, J. Rossmeisl, I.E.L. Stephens, Toward an active and stable catalyst for oxygen evolution in acidic media: Ti-stabilized $\mathrm{MnO}_{2}, \mathrm{Adv}$. Energy Mater. 5 (2015) 1500991.

[10] C.S. Mullins, V.L. Pecoraro, Reflections on small molecule manganese models that seek to mimic photosynthetic water oxidation chemistry, Coord. Chem. Rev. 252 (2008) 416-443.

[11] C. Zhang, C. Chen, H. Dong, J.-R. Shen, H. Dau, J. Zhao, A synthetic $\mathrm{Mn}_{4} \mathrm{Ca}$-cluster mimicking the oxygen-evolving center of photosynthesis, Science 348 (2015) 690-693.

[12] K. Fominykh, P. Chernev, I. Zaharieva, J. Sicklinger, G. Stefanic, M. Döblinger, A. Müller, A. Pokharel, S. Böcklein, C. Scheu, T. Bein, D. Fattakhova-Rohlfing, Iron-doped nickel oxide nanocrystals as highly efficient electrocatalysts for alkaline water splitting, ACS Nano 9 (2015) 5180-5188.

[13] H.C. Sansom, G.F.S. Whitehead, M.S. Dyer, M. Zanella, T.D. Manning, M.J. Pitcher, T.J. Whittles, V.R. Dhanak, J. Alaria, J.B. Claridge, M.J. Rosseinsky, AgBiI 4 as a lead-free solar absorber with potential application in photovoltaics, Chem. Mater. 29 (2017) 1538-1549.

[14] A.D. Handoko, S. Deng, Y. Deng, A.W.F. Cheng, K.W. Chan, H.R. Tan, Y. Pan, E.S. Tok, C.H. Sow, B.S. Yeo, Enhanced activity of $\mathrm{H}_{2} \mathrm{O}_{2}$-treated copper(II) oxide nanostructures for the electrochemical evolution of oxygen, Catal. Sci. Technol. 6 (2016) 269-274.

[15] X. Li, X. Hao, A. Abudula, G. Guan, Nanostructured catalysts for electrochemical water splitting: current state and prospects, J. Mater. Chem. A 4 (2016) 11973-12000.

[16] B. Li, F. Li, S. Bai, Z. Wang, L. Sun, Q. Yang, C. Li, Oxygen evolution from water oxidation on molecular catalysts confined in the nanocages of mesoporous silicas, Energy Environ. Sci. 5 (2012) 8229-8233.

[17] A.D. Doyle, J.H. Montoya, A. Vojvodic, Improving oxygen electrochemistry through nanoscopic confinement, ChemCatChem 7 (2015) 738-742.

[18] J.D. Blakemore, R.H. Crabtree, G.W. Brudvig, Molecular catalysts for water oxidation, Chem. Rev. 115 (2015) 12974-13005.

[19] T.E. Rosser, M.A. Gross, Y.-H. Lai, E. Reisner, Precious-metal free photoelectrochemical water splitting with immobilised molecular Ni and Fe redox catalysts, Chem. Sci. 7 (2016) 
4024-4035.

[20] M. Blasco-Ahicart, J. Soriano-López, J.R. Galán-Mascarós, Conducting organic polymer electrodes with embedded polyoxometalate catalysts for water splitting, ChemElectroChem (2017) doi:10.1002/celc.201700696.

[21] M.E.G. Lyons, R.L. Doyle, M.P. Browne, I.J. Godwin, A.A.S. Rovetta, Recent developments in electrochemical water oxidation, Curr. Opin. Electrochem. 1 (2017) 4045 .

[22] S. Hammes-Schiffer, Proton-coupled electron transfer: Moving together and charging forward, J. Am. Chem. Soc. 137 (2015) 8860-8871.

[23] C. Costentin, J.-M. Savéant, Theoretical and mechanistic aspects of proton-coupled electron transfer in electrochemistry, Curr. Opin. Electrochem. 1 (2017) 104-109.

[24] H. Lei, A. Han, F. Li, M. Zhang, Y. Han, P. Du, W. Lai, R. Cao, Electrochemical, spectroscopic and theoretical studies of a simple bifunctional cobalt corrole catalyst for oxygen evolution and hydrogen production, Phys. Chem. Chem. Phys. 16 (2014) 18831893.

[25] F. Acuña-Parés, Z. Codolà, M. Costas, J.M. Luis, J. Lloret-Fillol, Unraveling the mechanism of water oxidation catalyzed by nonheme iron complexes, Chem. Eur. J. 20 (2014) 5696-5707.

[26] D.W. Crandell, S. Xu, J.M. Smith, M.-H. Baik, Intramolecular oxyl radical coupling promotes $\mathrm{O}-\mathrm{O}$ bond formation in a homogeneous mononuclear Mn-based water oxidation catalyst: A computational mechanistic investigation, Inorg. Chem. 56 (2017) 4435-4445.

[27] A.V. Marenich, J. Ho, M.L. Coote, C.J. Cramer, D.G. Truhlar, Computational electrochemistry: prediction of liquid-phase reduction potentials, Phys. Chem. Chem. Phys. 16 (2014) 15068-15106.

[28] D.W. Shaffer, Y. Xie, J.J. Concepcion, O-O bond formation in ruthenium-catalyzed water oxidation: single-site nucleophilic attack vs. O-O radical coupling, Chem. Soc. Rev. (2017) doi:10.1039/C7CS00542C.

[29] P.E.M. Siegbahn, Nucleophilic water attack is not a possible mechanism for O-O bond formation in photosystem II, Proc. Natl. Acad. Sci. U.S.A. 114 (2017) 4966-4968.

[30] P. Miró, M.Z. Ertem, L. Gagliardi, C.J. Cramer, Quantum chemical characterization of water oxidation catalysts, in: A. Llobet (Ed.), Molecular Water Oxidation Catalysis: A Key Topic for New Sustainable Energy Conversion Schemes, John Wiley \& Sons, Ltd., United Kingdom, 2014, pp.233-255.

[31*] J. Soriano-López, D.G. Musaev, C.L. Hill, J.R. Galán-Mascarós, J.J. Carbó, J.M. Poblet, Tetracobalt-polyoxometalate catalysts for water oxidation: Key mechanistic details, J. Catal. 350 (2017) 56-63.

Thorough mechanistic study of Co-containing polyoxometalate clusters and the effect of its heteroatom on the OER activity. 
[32] V. Tripković, E. Skúlason, S. Siahrostami, J.K. Nørskov, J. Rossmeisl, The oxygen reduction reaction mechanism on $\operatorname{Pt}(111)$ from density functional theory calculations, Electrochim. Acta (2010) 7975-7981.

[33] J.K. Nørskov, J. Rossmeisl, A. Logadottir, L. Lindqvist, J.R. Kitchin, T. Bligaard, H. Jónsson, Origin of the overpotential for oxygen reduction at a fuel-cell cathode, J. Phys. Chem. B 108 (2004) 17886-17892.

[34] Y.-H. Fang, Z.-P. Liu, Mechanism and tafel lines of electro-oxidation of water to oxygen on $\mathrm{RuO}_{2}(110)$, J. Am. Chem. Soc. 132 (2010) 18214-18222.

[35] Y.-F. Li, A. Selloni, Mechanism and activity of water oxidation on selected surfaces of pure and Fe-doped $\mathrm{NiO}_{\mathrm{x}}$, ACS Catal. 4 (2014) 1148-1153.

[36] A.J. Tkalych, H.L. Zhuang, E.A. Carter, A density functional + U Aassessment of oxygen evolution reaction mechanisms on $\beta$-NiOOH, ACS Catal. 7 (2017) 5329-5339.

[37] A. Grimaud, O. Diaz-Morales, B. Han, W.T. Hong, Y.-L. Lee, L. Giordano, K.A. Stoerzinger, M.T.M. Koper, Y. Shao-Horn, Activating lattice oxygen redox reactions in metal oxides to catalyse oxygen evolution, Nat. Chem. 9 (2017) 457-465.

[38] J. Rossmeisl, E. Skúlason, M.E. Björketun, V. Tripkovic, J.K. Nørskov, Modeling the electrified solid-liquid interface, Chem. Phys. Lett. 466 (2008) 68-71.

[39] E. Skúlason, V. Tripkovic, M.E. Björketun, S. Gudmundsdóttir, G. Karlberg, J. Rossmeisl, T. Bligaard, H. Jónsson, J.K. Nørskov, Modeling the electrochemical hydrogen oxidation and evolution reactions on the basis of density functional theory calculations, J. Phys. Chem. C 114 (2010) 18182-18197.

[40**]K. Chan, J.K. Nørskov, Electrochemical barriers made simple, J. Phys. Chem. Lett. 6 (2015) 2663-2668.

Development of a new method for the calculation of electrochemical barriers at constant potential based on a "charge-extrapolation" scheme.

[41] K. Chan, J.K. Nørskov, Potential dependence of electrochemical barriers from ab initio calculations, J. Phys. Chem. Lett. 7 (2016) 1686-1690.

[42] K. Letchworth-Weaver, T.A. Arias, Joint density functional theory of the electrodeelectrolyte interface: Application to fixed electrode potentials, interfacial capacitances, and potentials of zero charge, Phys. Rev. B 86 (2012) 075140.

[43] Y. Ping, R.J. Nielsen, W.A. Goddard III, The reaction mechanism with free energy barriers at constant potentials for the oxygen evolution reaction at the $\mathrm{IrO}_{2}$ (110) surface, J. Am. Chem. Soc. 139 (2017) 149-155.

[44] J. Rossmeisl, A. Logadottir, J.K. Nørskov, Electrolysis of water on (oxidized) metal surfaces, Chem. Phys. 319 (2005) 178-184.

[45] J. Rossmeisl, Z.-W. Qu, H. Zhu, G.-J. Kroes, J.K. Nørskov, Electrolysis of water on oxide surfaces, J. Electroanal. Chem. 607 (2007) 83-89.

[46] M.T.M. Koper, Thermodynamic theory of multi-electron transfer reactions: Implications 
for electrocatalysis, J. Electroanal. Chem. 660 (2011) 254-260.

[47] I.C. Man, H.-Y. Su, F. Calle-Vallejo, H.A. Hansen, J.I. Martínez, N.G. Inoglu, J. Kitchin, T.F. Jaramillo, J.K. Nørskov, J. Rossmeisl, Universality in oxygen evolution electrocatalysis on oxide surfaces, ChemCatChem 3 (2011) 1159-1165.

[48*] W.T. Hong, R.E. Welsch, Y. Shao-Horn, Descriptors of oxygen-evolution activity for oxides: A statistical evaluation, J. Phys. Chem. C 120 (2016) 78-86.

Study and classification of 14 different chemical descriptors by means of a quantitative structureactivity relationships.

[49] M.G. Mavros, J.J. Shepherd, T. Tsuchimochi, A.R. McIsaac, T. Van Voorhis, Computational design principles of two-center first-row Ttransition metal oxide oxygen evolution catalysts, J. Phys. Chem. C 121 (2017) 15665-15674.

[50] J. Zaffran, M.C. Toroker, Metal-oxygen bond ionicity as an efficient descriptor for doped $\mathrm{NiOOH}$ photocatalytic activity, ChemPhysChem 17 (2016) 1630-1636.

[51] K. Toyoda, R. Hinogami, N. Miyata, M. Aizawa, Calculated descriptors of catalytic activity for water electrolysis anode: Application to delafossite oxides, J. Phys. Chem. C 119 (2015) $6495-6501$.

[52] Z. Zhao, Z. Xia, Design principles for dual-element-doped carbon nanomaterials as efficient bifunctional catalysts for oxygen reduction and evolution reactions, ACS Catal. 6 (2016) $1553-1558$.

[53] J. Tomasi, B. Mennucci, R. Cammi, Quantum mechanical continuum solvation models, Chem. Rev. 105 (2005) 2999-3093.

[54] B. Mennucci, Polarizable continuum model, WIREs Comput. Mol. Sci. 2 (2012) 386-404.

[55] K. Mathew, R. Sundararaman, K. Letchworth-Weaver, T.A. Arias, R.G. Hennig, Implicit solvation model for density-functional study of nanocrystal surfaces and reaction pathways, J. Chem. Phys. 140 (2014) 084106.

[56*] K. Mathew, R.G. Hennig, Implicit self-consistent description of electrolyte in plane-wave density-functional theory, (2016), http://arxiv.org/abs/1601.03346.

Implicit solvation model developed for the description of electrode/electrolyte interfaces and electrochemical reactions.

[57*] M. Garcia-Ratés, N. López, Multigrid-based methodology for implicit solvation models in periodic DFT, J. Chem. Theory Comput. 12 (2016) 1331-1341.

Development of a fast and efficient implicit solvation model for periodic DFT calculations.

[58] R. Sundararaman, K. Schwarz, Evaluating continuum solvation models for the electrodeelectrolyte interface: Challenges and strategies for improvement, J. Chem. Phys. 146 (2017) 084111.

[59] S. Siahrostami, A. Vojvodic, Influence of adsorbed water on the oxygen evolution reaction on oxides, J. Phys. Chem. C 119 (2015) 1032-1037. 
[60] J.A. Gauthier, C.F. Dickens, L.D. Chen, A.D. Doyle, J.K. Nørskov, Solvation effects for oxygen evolution reaction catalysis on $\mathrm{IrO}_{2}(110)$, J. Phys. Chem. C 121 (2017) 1145511463.

[61] D. Friebel, M.W. Louie, M. Bajdich, K.E. Sanwald, Y. Cai, A.M. Wise, M.-J. Cheng, D. Sokaras, T.-C. Weng, R. Alonso-Mori, R.C. Davis, J.R. Bargar, J.K. Nørskov, A. Nilsson, A.T. Bell, Identification of highly active $\mathrm{Fe}$ sites in $(\mathrm{Ni}, \mathrm{Fe}) \mathrm{OOH}$ for electrocatalytic water splitting, J. Am. Chem. Soc. 137 (2015) 1305-1313.

[62] E. Watanabe, J. Rossmeisl, M.E. Björketun, H. Ushiyama, K. Yamashita, Atomic-scale analysis of the $\mathrm{RuO}_{2}$ /water interface under electrochemical conditions, J. Phys. Chem. C 120 (2016) 8096-8103.

[63**]B. Zhang, X. Zheng, O. Voznyy, R. Comin, M. Bajdich, M. García-Melchor, L. Han, J. Xu, M. Liu, L. Zheng, F.P. García de Arquer, C.T. Dinh, F. Fan, M. Yuan, E. Yassitepe, N. Chen, T. Regier, P. Liu, Y. Li, P. De Luna, A. Janmohamed, H.L. Xin, H. Yang, A. Vojvodic, E.H. Sargent, Homogeneously dispersed multimetal oxygen-evolving catalysts, Science 352 (2016) 333-337.

Rational design of a ternary Fe,W-doped $\mathrm{CoOOH}$ catalyst exhibiting a record OER overpotential in alkaline solution.

[64**]J.W.D. Ng, M. García-Melchor, M. Bajdich, P. Chakthranont, C. Kirk, A. Vojvodic, T.F. Jaramillo, Gold-supported cerium-doped $\mathrm{NiO}_{\mathrm{x}}$ catalysts for water oxidation, Nat. Energy 1 (2016) 16053.

Calculations shed light on the electronic and geometric effects responsible for the observed enhanced catalytic of a Au-supported $\mathrm{NiCeO}_{x}$ catalyst.

[65*] Z. Lu, G. Chen, Y. Li, H. Wang, J. Xie, L. Liao, C. Liu, Y. Liu, T. Wu, Y. Li, A.C. Luntz, M. Bajdich, Y. Cui, Identifying the active surfaces of electrochemically tuned $\mathrm{LiCoO}_{2}$ for oxygen evolution reaction, J. Am. Chem. Soc. 139 (2017) 6270-6276.

DFT study on the elucidation of the catalyticaly active phase of $\mathrm{LiCoO}_{2}$ and the effect of delithiation in the OER performance.

[66**]L.C. Seitz, C.F. Dickens, K. Nishio, Y. Hikita, J. Montoya, A. Doyle, C. Kirk, A. Vojvodic, H.Y. Hwang, J.K. Nørskov, T.F. Jaramillo, A highly active and stable $\mathrm{IrO}_{\mathrm{x}} / \mathrm{SrIrO}_{3}$ catalyst for the oxygen evolution reaction, Science 353 (2016) 1011-1014.

Identification of meta-stable anatase $\mathrm{IrO}_{\mathrm{x}}$ as the OER catalytic active phase after leaching of $\mathrm{Sr}^{2+}$ from a $\mathrm{SrIrO}_{3}$ thin film.

[67*] M. García-Melchor, L. Vilella, N. López, A. Vojvodic, Computationally probing the performance of hybrid, heterogeneous, and homogeneous iridium-based catalysts for water oxidation, ChemCatChem 8 (2016) 1792-1798.

DFT calculations revealed that the design of hybrid electrocatalysts with the robustness of the heterogeneous materials and the high OER activity of homogeneous catalysts might be feasible. 